\title{
The interplay of drought and dehydration with the duration of plant growth: Application of bioinformatics for candidate gene identification in bread wheat
}

\author{
L. Zotova ${ }^{1 *}$, S. Jatayev ${ }^{1}$, V. Shvidchenko ${ }^{1}$, Y. Shavrukov ${ }^{2}$ \\ ${ }^{1}$ S. Seifullin Kazakh AgroTechnical University, Astana, Kazakhstan \\ ${ }^{2}$ Flinders University, Adelaide, Australia \\ *e-mail:lupezo_83@mail.ru
}

Key words: plant genetics, wheat, drought, abiotic stress, transcription factors

Introduction and Aim: Drought can affect plants at any stage of ontogenesis. Lateand early-maturing cultivars of wheat differ in their development in ways that can advantage grain production in the unpredictable occurrence of rainfall or drought. In the environment of Northern Kazakhstan, late-maturing genotypes of wheat are preferable in the absence of rainfall during the spring/early-summer season. In contrast, higher grain yield is typically shown by wheat genotypes with an early-maturing habit in conditions of terminal drought. Therefore, high grain yield represents an integrative trait, determined by not only yield potential but also the length of plant growth and reactions to drought. The aims of this study were to identify and analyse the most suitable gene candidates among Transcription factors regulating plant reactions to drought during the development of wheat plants and to carry out comparative bioinformatic analysis of similar genes in other plant species.

Methods: In this study, grain yield among an International germplasm collection of 200 bread wheats was studied in field trails in the Akmola region of Northern Kazakhstan as well as in laboratory treatments for drought and dehydration. Bioinformatics and systems biology approaches were used to identify and analyse the most important candidate genes during plant growth for higher grain yield.

Results: Two genes encoding different Transcription factors, TaDREB5 (Drought Responsive Element Binding) and TaNFYC-A7 (Nuclear Factor Y), showed the highest levels of gene expression in plants exposed to drought and dehydration, and were strongly associated with higher grain yield compared to controls. Similar results in other plant species in response to abiotic stresses were found and confirmed through bioinformatics analysis.

Conclusion: The results support the use of the above candidate genes for advanced selection of bread wheat genotypes for high grain yield with improved tolerance to drought and dehydration through adaptation to the required length of plant growth.

Acknowledgements: This study was supported by the Ministry of Education and Science. Kazakhstan, Research program BR05236500. 